\title{
Histological Study of Respiratory Organ of Betta sp.
}

\author{
Nurul Safitri Apriliani ${ }^{1,2 *}$, Hikmah Supriyati ${ }^{3}$, M. Ja'far Luthfi ${ }^{3}$ \\ ${ }^{1}$ Center for Integrative Zoology, ${ }^{2}$ Biology Department, ${ }^{3}$ Biology Education Department, Faculty of Science and Technology, UIN Sunan Kalijaga \\ Jl. Marsda Adisucipto No. 1 Yogyakarta 55281, Indonesia. Tel. + 62-274-540971, Fax. + 62-274-519739 \\ ${ }^{1}$ Email: nurulapriliani89@gmail.com
}

\begin{abstract}
Betta sp. is a freshwater ornamental fish which also known as a fighting fish. One of the fundamental organs to support fishes life is respiratory organ. Fighting fish is belongs to the suborder Anabantoidei which means labyrinth fishes group. The aim of the study was to know histology of the respiratory organs of Betta sp. Histological preparations were done using paraffin method, stained with Hematoxylin-Eosin (HE). The result showed that Betta sp. has a respiratory organ common fish i.e gills and additional respiratory organ structure namely labyrinth and pseudobranch that makes Betta sp can survive in a low volume of water. The gill is consists of gill arch, gill raker, gill fillament and gill lamellae. The labyrinth is consist of connective tissue and folded ephitelium. Pseudobranch according to some literature function as an additional respiratory. Functions attributed to the pseudobranch include; regulation of oxygen to the eyes, enzyme production for use in the gas bladder, osmoregulation, and many others.
\end{abstract}

Keywords: Betta sp., Fighting fish, Histology, Labyrinth, Pseudobranch, Respiration

\section{INTRODUCTION}

Betta sp. is a freshwater fish that has a special character, such as its aggressiveness to hold their territory from their fellow. Betta sp. has high survivor rates in a low volume of water with no air circulator (aerator). The fundamental organ to support the fish in that environment is respiratory system organ.

Respiration is a physiological process by which organisms exchange gases (oxygen and carbon dioxide) from the environment. It is taking place between blood and water through the medium of respiratory. This process is also known as an internal respiration process. The organ who take a respiratory system work as well is gill and other additional structure respiratory.

The gills of some fish species can compensate for ambient oxygen changes by exhibiting morphological and functional plasticity that give the gill the ability to modify its structure. Previous studies reveal aquatic airbreathing fish with accessory air-breathing organ (the labyrinth organ).

Air-breathing fish are defined as fish that have the ability to exchange gases directly from the aerial environment, and these all have an accessory airbreathing organ (Graham, 1997). They are classified into amphibious and aquatic air-breathing fishes. The accessory air-breathing organs are alternative gas exchange organs that are found in many different tissues including the labyrinth organ, skin, lungs, respiratory gas bladders, digestive tracts and structures derived from buccal, pharyngeal and branchial cavities from several different phylogenetic lineages (Graham, 1997). The species in the Anabantoidei have the labyrinth organ as an accessory air-breathing organ and possess branchial and systemic circuits similar to a double-circuit circulatory system (Munshi et al., 1986; Olson et al., 1986).
The other characteristic of Betta sp. that enable them to survive in the low water volume is a respiratory modification in the form of an air chamber filled with plates or leaflets of respiratory epithelium located above the gills and functioning adjuncts to them in obtaining oxygen. This adaption and variations of it are not uncommon in organisms which have become adapted to water which is frequently low in oxygen content.

Until recently the classification for the Betta was Anabantidae. Anabantid fishes are found throughout southeastern Asia as far north as northern China and Korea and including the Philippines and the Malayan archipelago to, and though, southern and western Africa. The Asian and African forms are somewhat distinct and the African forms are not known. Genus Betta has Asiatic distribution. Some are widely distributed, but according to Smith (1945) Betta splendens appears to be found in the wild state only in Siam (Thailand). It may be that they were widely distributed artificially for possible mosquito control at an early date, making it difficult to accurately determine their natural range.

Labyrinth fish are endemic to freshwaters of Asia and Africa. In Asia, they are found throughout East, Southeast, and South Asia, especially but not exclusively in the warm, slow-flowing, low-oxygen waters. In Africa, significantly smaller numbers of labyrinth fish can be found in the southern half of the continent, with concentrate in the rainforest waters. The characteristics of the fish habitats are indicators of the size of the labyrinth organ, as the organ size is negatively correlated with the level of oxygen in the waters. Species native to low-oxygen waters are more likely to have larger and more complex labyrinth organs than species found in fast-flowing, oxygen-rich waters (Pinter, 1986). 
This paper aimed to investigated histological and functional changes in the respiratory organs (gills and additional organs/labyrinth) of the aquatic air-breathing fish Betta splendens. The purpose of this research is to know the histology of gill as main respiratory organ and to know histology of labyrinth and pseudobranch as an additional respiratory organ of Betta splendens.

\section{MATERIALS AND METHODS}

The animals used in this study were three Betta fishes obtained from Market fish, Balirejo, Yogyakarta. The material used were bouin, decal solution, toluene, xylol, paraffin, ethanol, hematoxylin-eosin, glycerin, and entellan. The equipment used in this study were dissection kit, paraffin tub, flacon bottles, oven paraffin, staining jar, cassettes, beaker $250 \mathrm{ml}$, and 50 $\mathrm{ml}$, measuring cup $100 \mathrm{ml}$, funnel, stirrer glass, iron clamp, slide warmer, balance, rotary microtome, microscope, glass slide, cover glass, and camera. The organ was sacrificed and fixed with bouin's solution. Decalcifying tissue were done with decal solution for histological process. Histological slides were made using paraffin method with Hematoxylin-Eosin (HE) staining.

\section{RESULTS AND DISCUSSION}

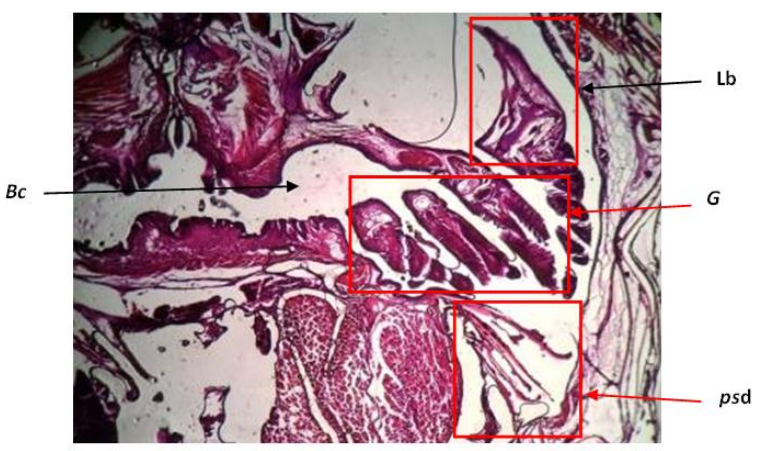

Figure 1. Histology of head part of Betta sp. (G) Gill; (Lb) Labyrinth; (psd) Pseudobranch; (Bc) Buccal cavity. HE staining. (4x10 Magnification).

Fish living in an environment that is considerably different from that encountered by terrestrial vertebrates. Compared with air, water is 800 times more dense, 60 times more viscous, has $1 / 30$ the capacity to hold oxygen and oxygen diffuses at $1 / 8000$ the rate. Thus this respiratory medium is more abrasive and oxygen depleted than air (Gary, 2000).

Most teleosts use gills as the main respiratory surface, although accessory respiratory structures also occur (Genten et al., 2009). The result showed that the histology of respiratory organ of fathead Betta sp. is consist of gill, labyrinth, and pseudobranch. In the aquatic environment, fish gills serve multiple functions, such as gas exchange, ionic regulation, acid-base balance, and nitrogen excretion, for the purpose of maintaining homeostasis (Perry, 1998; Evans et al., 2005).

The fish gill has become a multifunctional organ designed to deal with the vagaries of an aquatic medium. The gill is not only the primary site for respiration, it is also the principal, and often exclusive, site for osmoregulation, acid-base balance, and metabolism of circulating hormones and perhaps xenobiotics (Maetz, 1971; Hughes and Morgan, 1973; Heisler, 1984; Payan et al., 1984). The anatomy of gill tissue and its vasculature is as diverse and specialized as the function it performs and the gill might be the most highly differentiated of any vertebrate organ (Gary, 2000).

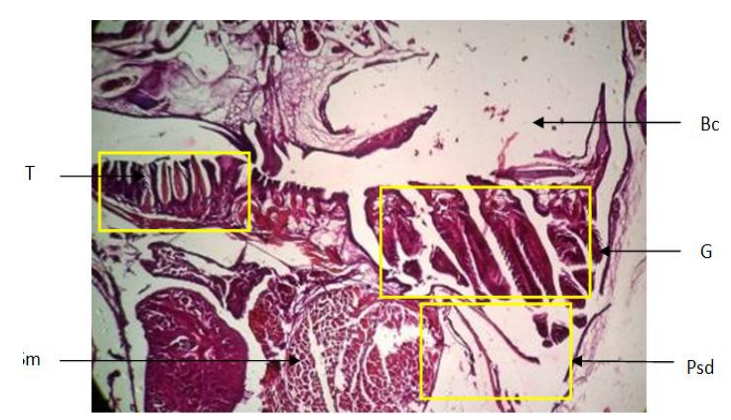

Figure 2. Histology of fathead of Betta sp. (Sm) Striated muscle; (T) Teeth; (Psd) Pseudobranch; (G) Gill; (Bc) Buccal cavity. HE staining. (10x10 Magnification).

Teleost fish have eight-gill arches arranged in four pairs on either side of the buccal cavity. An additional primordial gill hemiarch, the pseudobranch, is also present in most species, notable exceptions are the catfish. Structurally, the pseudobranch resembles a vestigial gill arch (Laurent and Dunel-Erb, 1984). It contains filaments similar to those on other gill arches, but they are generally covered by a relatively thick epithelium that prevents direct communication with the environment. Furthermore, the vascular supply to the pseudobranch is derived from the post-branchial (systemic) circulation (Gary, 2000).

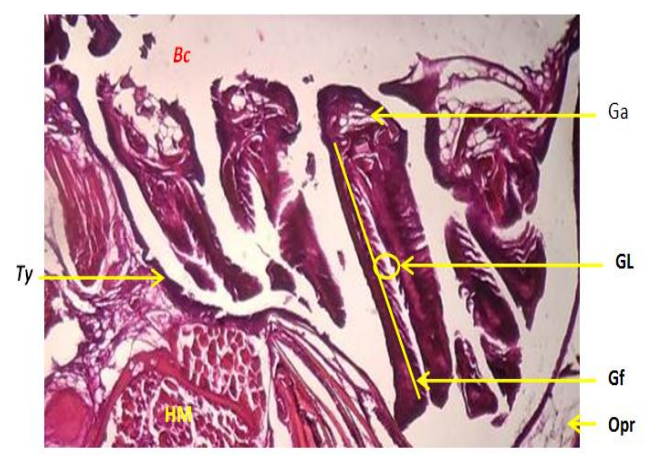

Figure 3. Histology of gill of Betta sp. (Ga) Gill arch; (Gf) Gill fillament; (GL) Gill lamellae; (HM) Hypaxial muscle; (Bc) Buccal cavity; (Ty) Thymus; (Opr) Operculum. HE staining. (10x10 Magnification). 
Most fish (teleost) have four pairs of gill arches extending from the floor to the roof of the buccal cavity. Each of the four pairs is supported by a cartilaginous and/or bony skeleton facilitating movement of gills to favorable respiratory positions. The gills are covered and protected by an operculum (Genten et al., 2009).

The four pairs of branchial arches in fish consist of many filaments and lamellae covered with epithelial cells. Basal wall, mitochondria-rich cells, mucous cells, and undifferentiated cells are the four major cell types in the gill epithelia (Perry, 1997; Evans, 1999). MRCs are generally distributed in the filaments and inter- and basal-lamellar regions and are believed to be the site of ionic extrusion in seawater fish and ionic uptake in freshwater fish (Perry, 1998; Evans, 1999; Evans et al., 2005; Hwang and Lee, 2007).

The inner surfaces of the gill arches carry one or more rows of stiff strainers called gill rakers. They serve to sort and aggregate particulate food material and to position larger food items before the food is passed into the esophagus and then into the stomach or intestine. The rakers tend to be long, slender, and tightly packed in planktivorous fishes and particle feeders (such as anchovies, herrings, alewife and certain scombrids) (Genten et al., 2009). Each gill raker is composed of an osseous or cartilaginous lamella supporting the pharyngeal pluristratified epithelium and connective tissue.

The purpose of the structures of gill lamellae is to provide a large surface area that supports respiratory and excretory functions. The efficiency of exchange, which in the case of oxygen is roughly $50-80 \%$, is largely a function of the countercurrent exchange between blood and water (Genten et al., 2009).

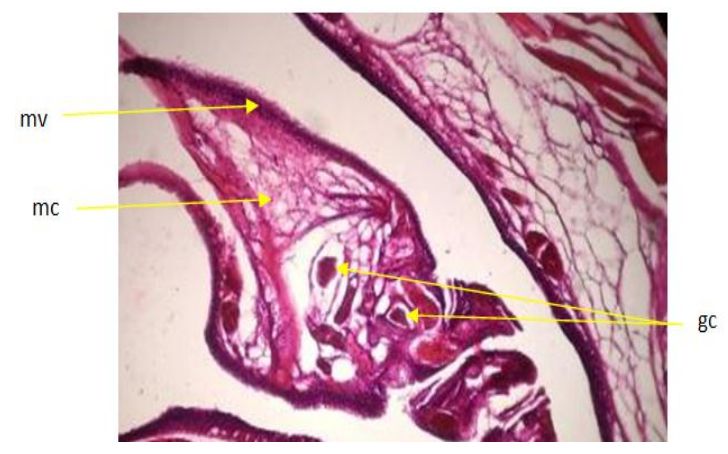

Figure 4. Histology of labyrinth of Betta sp. (mv) Microvilli; (mc) Mucous cell; (gc) Goblet cell. HE staining. (10x10 Magnification).

The labyrinth organ, a defining characteristic of fish in the suborder Anabantoidei, is a much-folded suprabranchial accessory breathing organ. It is formed by vascularized expansion of the epibranchial bone of the first-gill arch and used for respiration in the air (Pinter, 1986). This states is same with Munshi and Graham that Anabantoidei is aquatic-breathing fishes and have a labyrinth organ protruding from the first-gill arch on both sides of the branchial cavity to assist in gas exchange (Munshi et al., 1986; Graham, 1997).
This organ allows labyrinth fish to take oxygen directly from the air, instead of taking it from the water in which they reside through use of gills. The labyrinth organ helps the inhaled oxygen to be absorbed into the bloodstream. As a result, labyrinth fish can survive for a short period of time out of water, as they can inhale the air around them, provided they stay moist.

Labyrinth in fishes is not initially functional at immature individual (or at early life). The development of the organ is gradual and most labyrinth fish breathe entirely with their gills and develop the labyrinth organs later in life (Pinter, 1986).

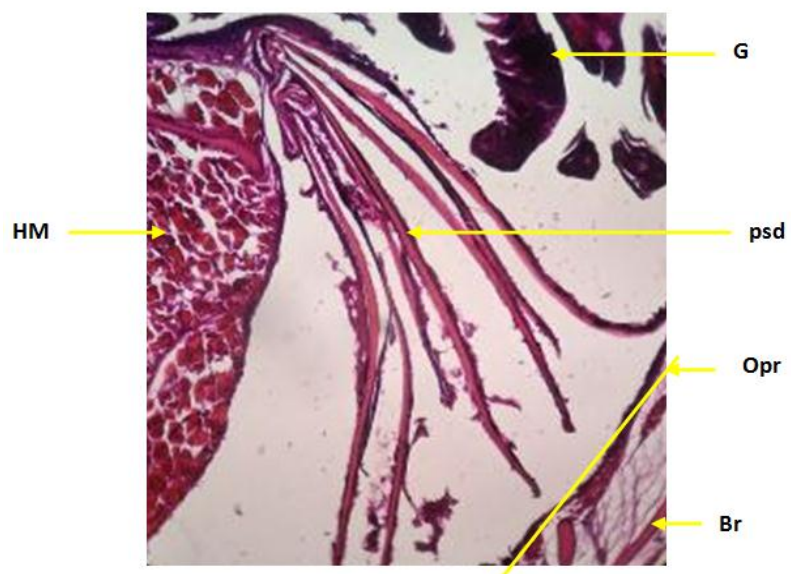

Figure 5. Histology of Pseudobranch (psd) of Betta sp. (G) Gill; (HM) Hypaxial muscle; (Opr) Operculum; (Br) Branchiostegal rays. HE staining. (10x10 Magnification).

The pseudobranch is a red, gill-like structure derived from the first-gill arch and located on the inner surface of the operculum. It is composed of gill lamellae, connective tissue, and blood vessels. The lamellae consist of pseudobranchial cells on an underlying basement membrane. This latter is applied to a network of parallel blood capillaries which can be supported by thin cartilaginous rods. The pseudobranch has a direct vascular connection with the choroid of the eye, which is composed of similar arrays of capillaries (rete mirabile) alternating with rows of fibroblast-like cells. The pseudobranch is not present in all teleosts. Those fish which do not possess such structure (some Siluridae, Ictaluridae, Notopteridae, Cobitidae, Anguillidae) invariably also lack a choroid rete. Although it is considered to have an endocrine and regulatory function as well as a hyperoxygenation function for the retinal blood supply, these are still to be defined in full.

In teleosts, pseudobranch occurs bilaterally along the interior of the opercula anterior to the first pair of gill arches. While morphologically similar to the gills, pseudobranchiae have a single row of filaments and receive oxygen-rich blood from the first efferent arteries (and are therefore unlikely to serve a respiratory function). In Cyprinidae, including Pimephales promelas, the pseudobranch is 
completely covered by the opercular epithelium. Lamellae, lacking any contact with the external medium, are fused to each other forming a "glandular pseudobranch" (Laurent and Dunel-Erb, 1984). The epithelium is comprised predominantly of pseudobranchial cells which are morphologically similar to chloride cells (but unique to the pseudobranch). Functions attributed to the pseudobranch include; larval respiration before maturation of gill arches, regulation of oxygen to the eyes, enzyme production for use in the gas bladder, osmoregulation, and many others. In a review of pseudobranch morphology and function, Laurent and Dunel-Erb (1984) found none of these explanations completely satisfactory, concluding that a likely function "should be at least partly sensory." These authors believe the pseudobranch, which is richly innervated, functions primarily in maintaining blood pressure.

Other from being assisted with labyrinth organ, Betta sp. also assisted by pseudobranch organ which has the same function to support respiration process in fishes. So the fishes can survive in lack of oxygen (environment). Pseudobranch only assisted in some species. In fact, Betta sp. is indeed have this pseudobranch. In some literature, the explanation about pseudobranch is still had the different meaning of the function. Other literature reported that pseudobranch functions to respiration, but the other explained that pseudobranch function to take oxygen into blood vessel to choroid eye.

\section{CONCLUSIONS}

Betta sp. as an ornamental fish has a special character, besides as a fighting fish, it has an ability to live in the low volume of water. It makes Betta sp. can survive in low-oxygen environment, as it has an additional respiratory organ (labyrinth, pseudobranch). Histologically, labyrinth is consist of connective tissue and folded epithelium. Pseudobranch according to some literature function as an additional respiratory. Functions attributed to the pseudobranch include; regulation of oxygen to the eyes, enzyme production for use in the gas bladder, osmoregulation, and many other.

\section{REFERENCES}

Evans DH, Piermarini PM, Choe KP. 2005. The multifunctional fish gill: dominant site of gas exchange, osmoregulation, acid-base regulation, and excretion of nitrogenous waste.Physiol Rev 85:97-177.

Gary K Ostrander. 2000. The laboratory Fish. Johns Hopkins University: Academic Press Baltimore MD, USA.

Genten F., Terwinghe E., Danguy A. 2009. Atlas Fish Histology. Science Publisher; USA.

Graham JB. 1997. Air-breathing fishes: evolution, diversity, and adaptation. New York, Academic Press.

Heisler, N. 1984. In Fish Physiology Vol X Gills, pp. 315

Huang CY, Lin CP, Lin HC. 2011. Morphological and biochemical variations in the gills of 12 aquatic airbreathing anabantoid fish Physiol Biochem Zool 84:125-134.

Hughes, G. M, Morgan, M. 1973. Biology. Rev, Academic Press, New York p48

Hwang PP, Lee TH. 2007. New insights into fish ion regulation and mitochondrion-rich cells. Comp Biochem Physiol 148A:479 497.

Laurent, P., Dunel-erb, S. 1984. In Fish Physiology. Vol X Gills. Part B: Ion and Water Transfer (eds W.W. Hoar and D. J Randall). Academic Press, New York.

Maetz, J. 1971. Phil. Trans. Roy. Soc. London. p 262.

Munshi JSD, Olson KR, Ojha J, Ghosh TK. 1986. Morphology and vascular anatomy of the accessory respiratory organs of the airbreathing Climbing perch, Anabas testudineus (Bloch). Am J Anat 176:321-331

Olson KR, Munshi JSD, Ghosh TK, Ojha J. 1986. Gill microcirculation of the air-breathing climbing perch, Anabas testudineus (Bloch): elationships with the accessory respiratory organs and systemic circulation. Am J Anat 176:305-320.

Payan, P, Girard, J. P.,, Mayer-Gostan, N. 1984. In Fish Physiology Vol X Gills. Academic Press, New York

Perry SF. 1997. The chloride cell: structure and function in the gills of freshwater fishes. Ann Rev Physiol 59:325-47.

Perry SF. 1998. Relationships between branchial chloride cells and gas transfer in freshwater fish. J Exp Biol 119:9-16.

Pinter, H. (1986). Labyrinth Fish. Barron's Educational Series, Inc 